\title{
SATISFACCIÓN RESIDENCIAL: UN CONCEPTO DE CALIDAD DE VIDA EN LA CIUDAD DE MONTREAL
}

\section{RESIDENTIAL SATISFACTION: A QUALITY OF LIFE CONCEPT IN MONTREAL CITY}

\author{
Carlos Alberto Tello Campos*
}

\section{RESUMEN}

El abandono de los sectores centrales y la incontrolable expansión de los periféricos en la ciudad de Montreal, es un fenómeno urbano de los siglos xx y xxi que atenta contra la satisfacción residencial y la calidad de vida local. Esta investigación realiza una encuesta en el sector central de la ciudad para evaluar ese problema en términos de la satisfacción e importancia residencial como índices subjetivo y objetivo de la calidad de vida (CvD) desde la perspectiva de un análisis de correlación lineal (SPSS).

PALABRAS CLAVE: CANADA * PLANIFICACIÓN URBANA * CALIDAD DE VIDA * CENTRO DE LA CIUDAD * PROGRAMAS DE VIVIENDA

\section{ABSTRACT}

The abandon of the central sectors and unbridled expansion of the peripheral ones in the city of Montreal is an urban phenomenon in xx and xxi centuries' that threatens the local residential satisfaction and quality of life. This research conducts on the central sector of the city a survey to evaluate that problem in terms of the residential satisfaction and importance as quality of life (QOL) subjective and objective indices from a linear correlation perspective (sPss).

KEYWORDS: CANADA * URBAN PLANNING * QUALITY OF LIFE * TOWN CENTRE * HOUSING PROGRAMME

Université de Montréal, Canadá.

carlos_alberto_tello@hotmail.com 


\section{INTRODUCCIÓN}

En el mundo, las áreas urbanas son los centros vitales para el desarrollo de la cultura y del gobierno, así como de muchos otros campos. Actualmente, las ciudades crecen a un ritmo dramático (intra-urbano e inter-urbano), despoblando a sus sectores centrales y poblando a los periféricos, lo que ocasiona que la población urbana total con más del $50 \%$ de la mundial alcance el $60 \%$ para el año 2030 (Demographia, abril 2009) pasando de 3000 a 5000 millones (United Nations, 2003). El despoblamiento central y la expansión periférica producen un cambio rápido y constante, tanto en la relación centro-periferia ${ }^{1}$ como en la frontera urbana-rural. Si bien, este desarrollo urbano a menudo conduce a graves problemas sociales, económicos y ambientales ${ }^{2}$ (progresiva gentrificación central y masiva expulsión de pobres, reducción de ingresos municipales, problemas de transporte centro-periferia, etc.), las cuales de racionalizarse pueden convertirse en una excelente oportunidad para convertirlo en sustentable. No obstante, lograr esto requerirá de un gran avance en la planificación, la tecnología y la forma de gobernar y administrar a las ciudades, a través de adecuados programas de renovación para preservar la calidad de vida (cDv) local.

Actualmente, los países norteamericanos están poniendo cada vez más atención a la sustentabilidad natural y material (social, económica y ambiental del contexto urbano) de sus áreas urbanas (United Nations, 1996) y más concretamente, al crecimiento centro-periferia de la ciudad (Sénecal, 1996).

En Canadá, Bourne, Hutton, Shearmur y Simmons (2011) y Tello (2013) han dirigido su atención al asunto del cambio en los gradientes de densidad $y$ en los diferentes grados

$1 \quad$ Según Solomon (2010) “... le jugamos a ser Dios al dirigir la migración local hacia áreas primordialmente agrícolas, sustituyendo nuestra mejor tierra arable por una expansión urbana sin control". The Urban Renaissance Institute, Toronto, Canada.

2 En el contexto de esta investigación, ambiente significa todo componente urbano construido o no por el hombre que forma parte integral de una ciudad. de centralidad de las ciudades canadienses. En ese sentido, sea en el caso de la centralidad primaria (centro) o secundaria (periferia), el proceso de crecimiento urbano (formal o informal) que se da simultáneamente al de la creación de una ciudad, se ha apoyado en la ilusión del acceso a vastos recursos en términos de tierras rurales y fuentes de energía "siempre disponibles" para la expansión horizontal. Si se considera que los recursos antes citados son de carácter limitado y bajo un criterio de economía urbana ciertamente no neoliberal, dicha expansión se vuelve más racional si tiende a respetar el principio de eficiencia ${ }^{3} y$ optimización del espacio existente (reciclado de espacios centrales) para apoyar la sustentabilidad. Algunos de los esfuerzos municipales se han enfocado a lograr ese objetivo. Agotar la disponibilidad del espacio existente antes de pasar a expansiones urbanas (invasiones) de tierra rural se ha reflejado sin duda, en acciones más sustentables centro-periferia que incrementa la compacidad de estructuras para controlar la extensión horizontal (complementariedad: re densificación-expansión) (Roseland, 1992).

Una encuesta de satisfacción e importancia residencial ha sido implementada en Montreal para investigar esta inquietud. Así, los esfuerzos de tipo sustentable para renovar vivienda $y$ servicios que se han instrumentado recientemente en el centro de la ciudad, han intentado recuperar los niveles de satisfacción residencial ${ }^{4}$ y calidad de vida del pasado; asimismo, con estos datos demográficos, realizar una significativa contribución en dicha tarea.

Este estudio analiza al abandono central y la incontrolable expansión de Montreal en el periodo más crítico de dicho proceso (19712006). Su selección como un caso de estudio de un fenómeno mundial, obedece a sus características particulares de crecimiento urbano bajo

$3 \quad$ Se entiende como eficiencia al nivel de cumplimiento de las metas cualitativas (satisfacción residencial) y cuantitativas (re-densificación) establecidas por cada programa de renovación.

4 Satisfacción residencial es el grado de resolución de necesidades habitacionales alcanzado por la vivienda en cuestión. 
criterios social, económico y ambiental que a continuación se detallan.

\section{CRECIMIENTO}

Un ejemplo representativo de dichas acciones de crecimiento urbano (despoblamiento central y expansión periférica) experimentado en el último cuarto del siglo $x x$, ha sido sin duda alguna la ciudad de Montreal en América del Norte.

En el contexto de las acciones de despoblamiento central, el sector central de Montreal compuesto por los municipios de Outremont, Westmount, Verdun y Montréal, demográficamente bajó de 1022630 habitantes en 1971, a solo 760177 en 1991 remontando a partir de ahí hasta llegar a una tendencia de estabilización de 775461 en 2001, en función de los programas de renovación implantados por el gobierno en dicho sector. La pérdida total de población en el punto demográficamente más bajo fue de 262453 residentes (25,7\% de la base original) (Statistics Canada, 1971, 1991 y 2001).

Por su parte, la zona metropolitana de la ciudad registró una población total de $1.8 \mathrm{mi}$ llones de habitantes en 1951, para alcanzar 3.6 en 2001 (Statistics Canada, 2001), participando los suburbios para 1990, con el 35\% del peso demográfico total (Roy y Weston, 1990). En cuanto a las expectativas urbanas, las extrapolaciones que específicamente se estimaron para este estudio en el sector central, indican que el umbral de análisis para el año 2026, todas las acciones de re-densificación que se emprendan no serán suficientes para reponer la población base de 1971. Para el 2026, en el sector central de Montreal, la recuperación demográfica llegará en la mejor de las situaciones, al 79,6\% del total original demográfico de 1971(Tello, 2008).

En ese contexto, la velocidad de crecimiento suburbano de la Zona Metropolitana de la ciudad de Montreal continuará a un ritmo significativo. Con base en este hecho, se prevé que el fenómeno de expansión periférica, sea este formal o informal, no disminuirá en un futuro próximo en Montreal, por lo que la desaparición de áreas rurales proseguirá en este caso. Por esta razón es importante dirigir todos los esfuerzos posibles para concentrarse en una mejor relación entre la sustentabilidad de los recursos disponibles, la población existente $y$ el uso de los niveles ecológicos y materiales para un determinado asentamiento humano. Sin una forma de planificación más consciente de esta relación en términos más eficientes, el abuso de recursos probablemente continuará su inexorable marcha con grandes impactos negativos sobre el medio ambiente, la satisfacción residencial y últimamente, la cDv local.

\section{RENOVACIÓN}

En el marco de una dinámica impulsada por la ciudad a fines de los años 70 para re-densificar al sector central, invirtiendo la secular tendencia de reducción demográfica local con el objetivo de estabilizar los impuestos prediales, algunos de los esfuerzos de renovación aplicados (vivienda social) adoptaron en general un carácter menos institucional al cambiar en años recientes el tradicional enfoque político de tipo "social" que los había caracterizado, por uno nuevo de "accesibilidad" (Affleck, 2004 y Tello, 1997). La idea detrás de dicho cambio fue de incrementar el nivel de atracción de los diferentes distritos que componen este sector, tanto para la inversión habitacional como para el consumidor de clase media. En lo particular, esos esfuerzos se han concentrado en incrementar la variedad en el régimen de ocupación residencial, al crear más oportunidades para la compra de propiedades en lo que ha sido llamado "la ciudad de los inquilinos" (Rose, 2004; Germain y Rose, 2000).

De esta manera, con el fin de apoyar la sustentabilidad de los recursos disponibles, se hicieron esfuerzos de renovación más representativos puestos en práctica a la fecha en Montreal y así limitar en lo posible, la decadencia urbana del centro de la ciudad, así como, el abuso de tierra rural en la periferia. Entre esos esfuerzos se encuentran: 1) "Vivienda Accesible Quebéc" (Logement Abordable Québec (LAQ): Ville de Montreal, 2005: 2, 3, 5, 7 y 8; Affleck, 2004: 17; Chantal, 2004: 7); 2) "Acceso-Vivienda" (Accès-Logis ( $\left.{ }_{A L}\right)$ : Chantal, 2004; Societé d'Habitation du Québec, 2005) y 3) "Renovación Quebéc" (Rénovation Québec 
$(R Q)$ : Rose, 2004: 18-19; Chantal y Charbonneau, 2004:1-2).

En ese contexto, este análisis de años 1971-2001 sobre el crecimiento habitacional, permite conocer que en el centro de la ciudad - particularmente, en aquellas regiones de los municipios de Verdún y Montreal que hasta 1996, se habían caracterizado por generar una decadencia urbana importante (por ejemplo en este último municipio, el perímetro II distritos de Ville-Marie/ Centre-Sud) (figura 1) - se había registrado un modesto aumento de unidades habitacionales del 2,4\% y $0,1 \%$ en Verdún, así como del 5,5\% y 5,6\% en Montreal para los años 1981-1991, 1991-2001, respectivamente (tabla 1). Esta situación es muy consistente con la tendencia estacionaria detectada en el área bajo estudio.

FIGURA 1

MONTREAL. ZONA DE ANÁLISIS: REGIONES DECADENTES, 1996

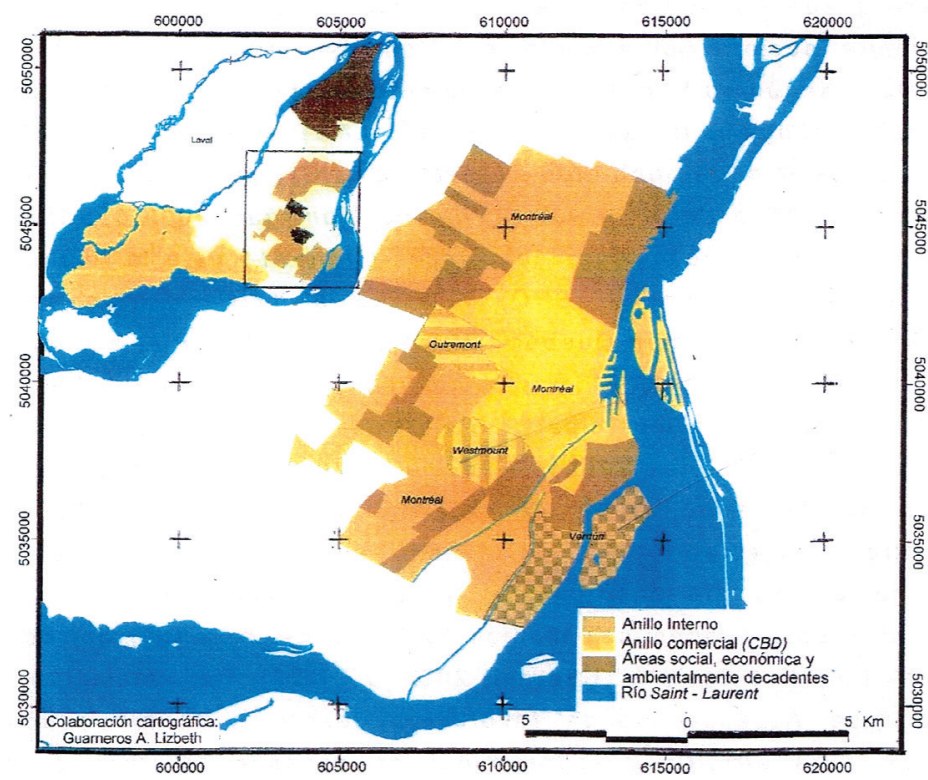

Fuente: Dansereau, Charbonneau, Morin, Revillard, Rose y Seguin, 2002; basado en Mongeau y Archambeault, 2001.

TABLA 1

CRECIMIENTO TOTAL EN EL SECTOR CENTRAL 1971-2001

\begin{tabular}{cccc}
\hline & $1971-1981$ & $1981-1991$ & $1991-2001$ \\
\hline Verdun $(1)$ & $-0,18 \%(-40)(3)$ & $+2,4 \%(545)(3)$ & $+0,1 \%(35)(3)$ \\
Montreal $(2)$ & $-0,04 \%(-120)(3)$ & $+5,5 \%(16735)(3)$ & $+5,6 \%(18020)(3)$ \\
\hline
\end{tabular}

Regiones en decadencia:

(1) Comprende Verdun.

(2) Comprende los siguientes distritos: Côte-des-Neiges/Notre-Dame-de-Grâce; Mercier/Hochelaga/Maisonneuve; Rosemont/Petite-Patrie; Sud-Ouest; Villeray-Parc Extension/St. Michel; Ville-Marie/Centre-Sud.

(3) Ganancias/pérdidas de viviendas a nivel municipio.

Fuente: Elaboración propia a partir de Statistics Canada, 1971, 1981, 1991 y 2001. 
El reciente impacto que las citadas políticas y programas tuvieron se hizo sentir en algunas partes del sector central al registrarse en el municipio de Montreal, un ligero aumento demográfico del 0,9\%, al pasar de 685393 habitantes en 2001 a 692000 en 2006, lo que ha afectado algunas de las áreas más habitadas de 2001 como en el perímetro II, a Ville-Marie/ Centre-Sud con 89830 habitantes (Statistics Canada, 2001).

Económicamente, el empleo municipal registró a 328655 personas con trabajo en 2001, destacando nuevamente el perímetro II, Ville-Marie/Centre-Sud con unas 42470 personas ocupadas (ibíd.). La generación local de 16 845 empleos, atribuidos solamente al nivel de sustitución de trabajos secundarios por terciarios (10 660) y cuaternarios (6 185) (un poco menos intenso en el distrito Centre-Sud que en el distrito de Ville-Marie), es un punto a considerar (ibíd.).

En cuanto al ambiente, los programas de renovación (vivienda) indicados se han hecho presentes en el municipio a través de los 19210 inicios de obra emprendidos en la construcción $y$ mantenimiento de inventario habitacional local, especialmente durante la segunda mitad del periodo 1994-2004 (Canada Mortgage and Housing Corporation (смнс), 2004). En ese contexto, en el perímetro II, los distritos VilleMarie/Centre-Sud señalados por la literatura (Statistics Canada, 1986 y 2001) como distritos con fuerte decadencia social, económica y ambiental, ocupan un destacado lugar en el proceso de mejoramiento del inventario habitacional local con 5242 inicios de obra (смнс, 2004).

Con respecto al inventario de vivienda social, Ville-Marie/Centre-Sud sobresalen con el mayor porcentaje de unidades (tabla 2), (Ville de Montréal, 2005: 2, 3, 5, 7 y 8). La construcción de este tipo de unidades habitacionales, en el periodo comprendido de 1999-2003, indica que el municipio de Montreal concentró en VilleMarie/Centre-Sud un porcentaje significativo de estas, así como en 2004, en relación al total de la oferta de vivienda local (ibíd.).

TABLA 2

INVENTARIO DE VIVIENDA SOCIAL EN EL SECTOR CENTRAL 2003

\begin{tabular}{lc}
\hline REGIONES EN DECADENCIA (1) & 2003 \\
\hline Municipio de Verdun & 1115 \\
\hline Municipio de Montréal & \\
\hline -Cote-des-Neiges/Notre-Dame-de-Grace & 4421 \\
-Mercier/Hochelaga /Maisonneuve & 3194 \\
-Rosemont/Petite-Patrie & 4478 \\
-Sud-Ouest & 6311 \\
-Villeray-Parc Extension/St. Michel & 3172 \\
-Ville-Marie/Centre-Sud & 7272 \\
\hline TOTAL & 29963 \\
\hline
\end{tabular}

(1) Comprende a los municipios y distritos que forman los perímetros I y II (fig. 1).

Fuente: Ville de Montreal, 2005.

De esta forma es posible constatar que Ville-Marie/Centre-Sud han sido los distritos centrales en donde se ha dado la creación de vivienda social y por ende, de renovación más significativa (Statistics Canada, 2006). En ese espacio geográfico, el barrio de Sainte Marie es un ejemplo representativo. A pesar de las acciones de mejoramiento tomadas, persiste todavía una decadencia social, económica y ambiental que ha fomentado una serie de problemas de indigencia, pobreza y degradación ambiental importante; todos estos puntos permanecen aún como importantes asuntos a resolver. 
En función a la renovación, el municipio de Montreal con el perímetro iI (el distrito de Ville-Marie/Centre-Sud), es entonces el espacio a estudiar debido a sus tendencias de crecimiento y mayor aplicación de los programas presentados: ¿en qué medida los programas de renovación aplicados en el municipio de Montreal-perímetro II (Ville-Marie/Centre-Sud) han sido eficientes vis-à-vis el nivel de satisfacción residencial lograda en términos de índices de correlación residencial? ${ }^{5}$

\section{OBJETIVOS DE INVESTIGACIÓN}

De la literatura científica disponible en Norteamérica, se resalta que Montreal experimentó a fines del siglo pasado un importante proceso de contracción demográfica en sus sectores centrales, aunado a una importante expansión en sus sectores periféricos. Esta realidad ha sido la razón principal para su selección como objeto de análisis de dicho proceso, en términos del impacto de esa realidad sobre la satisfacción residencial y calidad de vida.

Con base en esto, los objetivos de investigación son: 1) analizar a través de una encuesta, desde el punto de vista de las correlaciones de satisfacción/importancia con la vivienda (condición residencial) y servicios en los sectores centrales de Montreal, el impacto de la renovación urbana bajo el principio de desarrollo sustentable (optimización y reciclado de espacios) y 2) identificar las variables más significativas que determinan ese proceso de correlación vis-á-vis la satisfacción residencial con la condición de la vivienda y servicios (calidad de vida local). A partir de estos objetivos, surgen dos preguntas de investigación: a) ¿es la atracción urbana significativa en los sectores centrales de Montreal? y b) ¿qué tan eficiente ha sido la renovación urbana implantada para fomentar un desarrollo más compacto en la ciudad?

$5 \quad$ Correlación residencial se refiere a la intensidad con la cual dos variables (atributos) de una misma entidad habitacional o de servicios, se relacionan entre sí.

\section{HIPÓTESIS DE INVESTIGACIÓN}

En muchas ciudades de América del Norte como en el caso de Montreal, los programas de renovación urbana han sido lo suficientemente eficientes como para promover mayores correlaciones de satisfacción e importancia residencial en el sector central de la ciudad. Esta situación apoya más eficazmente a las iniciativas de re-densificación que los municipios respectivos conducen a la fecha.

\section{METODOLOGÍA DE INVESTIGACIÓN}

Considerando lo antes planteado dentro de un contexto de desarrollo sustentable (optimización de espacios, re-densificación y satisfacción residencial) se diseñó una metodología en dos etapas siguiendo un proceso de inducción.

Para alcanzar la sustentabilidad deseada en los desarrollos centrales de Montreal han intervenido distintos programas de renovación relativos al uso del suelo, superestructura (vivienda, etc.), infraestructura, oferta/demanda de transporte (viajes origen-destino) entre otros, con objeto de racionalizar el crecimiento centroperiferia de la ciudad (modelos multi-nucleares. Consultar Castells, 1969). A través de dicha renovación, ese crecimiento es el resultado directo de las fuerzas actuantes de atracción urbana, tanto sociales como económicas y ambientales.

Tomando en cuenta lo argumentado, los objetivos, las preguntas e hipótesis de investigación enunciadas encuentran una respuesta usando la metodología propuesta. En ese orden, en el área de estudio seleccionada (Municipio de Montreal-perímetro II), la revisión de la literatura científica más distintiva del periodo de estudio considerado, identificó distintas acciones de crecimiento central en función de diferentes atributos de atracción que establecieron una lista de variables (indicadores) sociales, económicas y ambientales a partir de censos de Statistics Canada, las cuales se trabajaron en dos etapas: a) recopilación de datos y b) procesamiento de datos (correlación lineal).

La revisión literaria se concentró en la consulta de censos canadienses y literatura diversa, período 1971-2006 para Montreal. En 
función de esto fue posible construir distintos escenarios de tipo social (demográfico), económico y ambiental para el perímetro señalado. El objeto fue el de centrar la atención en el desarrollo que la ciudad ha sostenido en ese período. Posteriormente, la selección de variables se apoyó en el criterio de sustentabilidad (variables que por su naturaleza son susceptibles de ser "recicladas" como las de vivienda).

Para tal fin, 86 variables fueron en un principio consideradas para el análisis hasta llegar al conjunto más significativo y explicativo, el cual fue agrupado en las categorías: a) contexto urbano primario: municipio, vivienda, servicios de apoyo; b) contexto urbano secundario: sitios de reunión, comercios y servicios privados, servicios públicos.

Por lo que corresponde en la "etapa a" a las actividades de recopilación de datos, tres niveles de representatividad de muestras en relación a su grado de desagregación fueron primeramente identificados, para de ahí seleccionar a la más conveniente en función de los recursos asignados al estudio: a) nivel manzana (block); b) nivel hogar y c) nivel residente. El nivel manzana se refiere al número de estas inscrito en el área de estudio. El nivel hogar se refiere al número de los mismos que se ubican dentro de dicha área. El nivel residente se refiere al número de personas que viven dentro de los límites del área. Esta investigación definió la representatividad de la muestra a nivel manzana.

El tamaño estadístico de la muestra analizada en el perímetro II se estimó calculando el número de manzanas que el perímetro contiene (aproximadamente 500) para con esto, definir con la asistencia de algunos tabuladores, los límites de confiabilidad superiores e inferiores en universos pequeños "N" (tables for upper and lower confidence bounds for small universes) (Wright, 1991), su tamaño "n", así como a los límites de confiabilidad.

Tomando en cuenta un muestreo al azar simple (simple random sampling approach) que en este sentido establece para cada elemento del universo considerado una misma probabilidad de ser seleccionado, así como a la población de dicho universo $\mathrm{N}=500$, los tabuladores determinaron que el número de unidades " $n$ " con un atributo particular "A" sea igual a $50(10 \%$ de
"N") (ibíd.: 332) en Montreal, para un nivel de confiabilidad de 95\% (ibíd.: 349). La confirmación posterior en Wilson y Morgan (2007: 4748) del tamaño encontrado confirma a "n" 50 como una muestra razonable para análisis de correlación. Una vez determinado el tamaño se realizó una encuesta para evaluar la eficiencia de la renovación implementada desde el punto de vista de la satisfacción e importancia residencial en los sitios indicados.

En la "etapa b", el procesamiento de los datos de campo y posterior construcción de índices se efectuó con técnicas de análisis multivariable usando el paquete estadístico SPSS (Babbie, 1993). En ese paquete, se escogió como herramienta de análisis a la correlación lineal debido a su gran poder de explicación claro y preciso del grado de interrelación biunívoca que las variables seleccionadas guardan en términos de dirección y magnitud. Dicha explicación llega de esta forma a ser fácil de visualizar para así lograr una mejor comprensión de la situación a través de los índices correspondientes, en la que el fenómeno urbano objeto del presente estudio se encuentra.

\section{CORRELACIÓN RESIDENCIAL}

La correlación lineal de la matriz de datos, producto de la investigación de campo en el sector central de la ciudad de Montreal, perímetro II (figura 2), se basó en los niveles de frecuencias biunívocas y de significación obtenidos. Entre las variables con índices más notables, destacaron las relacionadas con los citados contexto urbano primario ${ }^{6}$ (vivienda) y secundario $^{7}$ (sitios de reunión, servicios públicos).

$6 \quad$ Contexto urbano primario constituido a saber por el Municipio (variables-seguridad, tranquilidad, mantenimiento de propiedades, calidad del aire, áreas verdes); la Vivienda (variables-condición, tamaño, mantenimiento, confort); los Servicios de Apoyo (variables-bomberos, policía, recolección de basura, mantenimiento de calles $y$ banquetas, calidad del agua).

7 Contexto urbano secundario constituido a saber por Sitios de Reunión (variables-lugares socioculturales, deportivos, recreativos); Comercios y Servicios Privados (variables-tiendas de abarrotes, supermercados, farmacias, tlapalerías, bancos, restoranes, boutiques); Servicios Públicos (variables-centros de ayuda social, hospitales, escuelas, transporte, parques). 
FIGURA 2

MONTREAL. PERÍMETROS I Y II. SECTOR CENTRAL

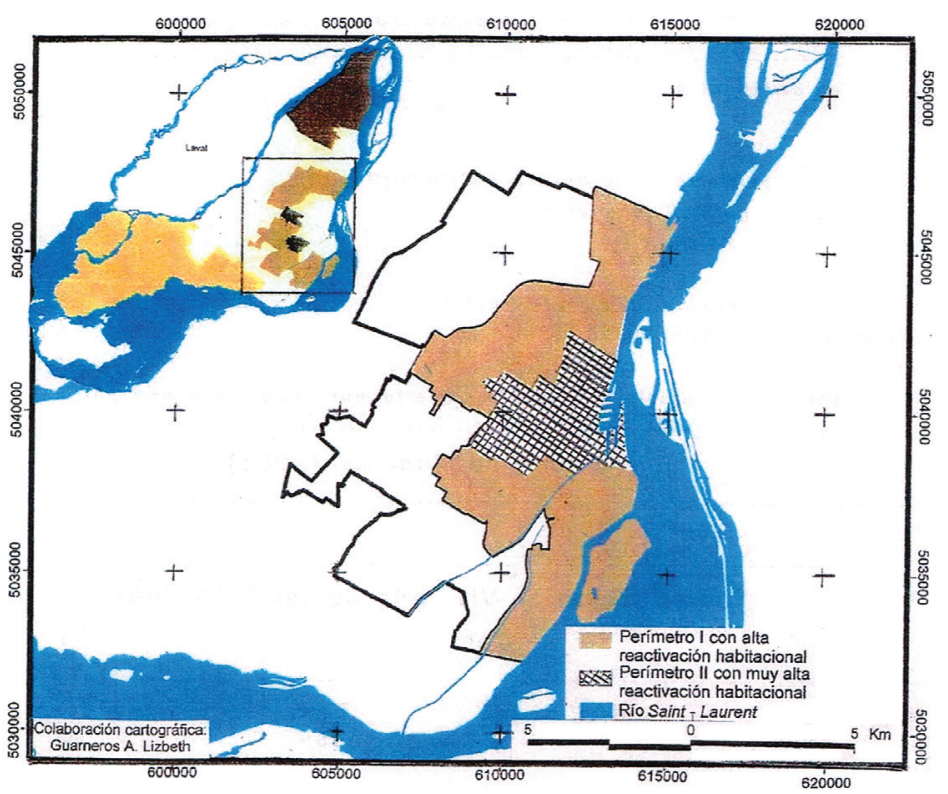

Fuente: Ville de Montréal, direction de l'habitation, 2002

En el primario, los grados de satisfacción (señalado en la literatura científica como atributo principal del concepto de calidad de vida o cDv) e importancia (atributo principal del concepto de calidad de lugar $0 \mathrm{cDL}$ ) acordados a la condición (proxy del proceso de renovación), tamaño, mantenimiento y confort de la vivienda, así como en el secundario en relación a la satisfacción e importancia acordadas a los lugares socioculturales $y$ recreativos de sitios de reunión y a los centros de ayuda social y hospitales de servicios públicos, fueron críticos para evaluar el estado actual de satisfacción residencial.

\section{ANÁLISIS}

En el contexto urbano primario del municipio de Montreal-perímetro II, rubro vivienda, los programas de renovación urbana canadienses exponen la gran atención que le han dedicado a la fecha, al cuidado del hábitat según los resultados de correlación lineal presentados en términos de la satisfacción residencial.

Esa atención es evidente al considerar la evaluación de la satisfacción sentida con la condición de sus viviendas en función de la equivalente con su mantenimiento reportada por la población residente del área (tabla 3 , gráfico 1 ). 
TABLA 3

CORRELACIÓN RESIDENCIAL

MONTREAL

\begin{tabular}{cc}
\hline Satisfacción con condición / satisfacción con mantenimiento & 0,94 \\
Satisfacción con tamaño / satisfacción con confort & 0,83 \\
Satisfacción-mantenimiento/ satisfacción con confort & 0,85 \\
Importancia por condición / importancia por mantenimiento & 0,95 \\
Importancia por tamaño / importancia por confort & 0,83 \\
Importancia-mantenimiento / importancia por confort & 0,92 \\
Sat.-lugares socioculturales/sat. lugares recreativos & 0,99 \\
Importancia-ctos ayuda soc./importancia por hospitales & 0,93 \\
\hline
\end{tabular}

Fuente: Elaboración propia.

\section{GRÁFICO 1 \\ CORRELACIÓN RESIDENCIAL \\ MONTREAL}

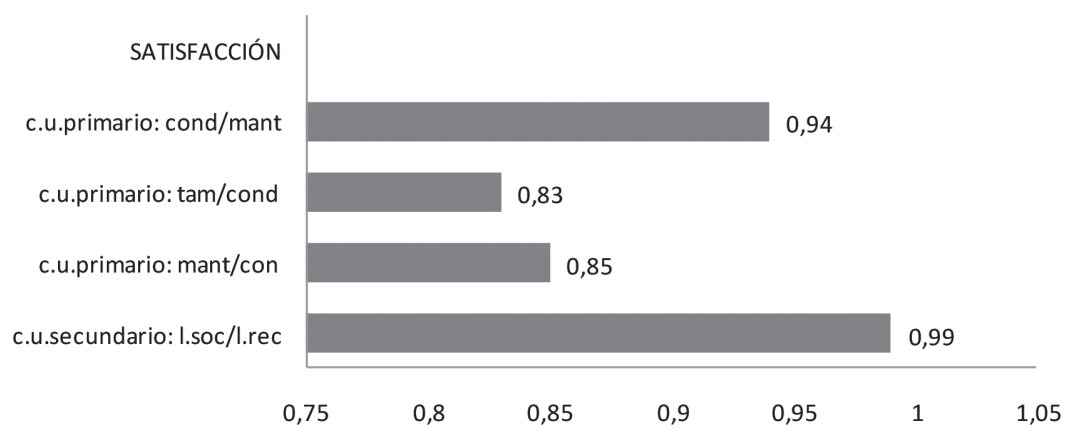

Fuente: Elaboración propia.

Con un índice muy alto de 0,94 , los residentes expresan así su opinión. La satisfacción con la condición de la vivienda es determinada en un $94 \%$ por la satisfacción con el mantenimiento cambiando así, en dirección y magnitud, una variable con respecto a la otra (una mayor satisfacción con la condición de la vivienda, es explicada por medio de un mayor mantenimiento invertido).

En cuanto a la satisfacción con el tamaño de las viviendas desde el ángulo del confort, los habitantes del perímetro tratado reportan sentirse aceptablemente bien (tabla 3, gráfico 1). El análisis de correlación de las variables ha producido un índice de 0,83 , lo que representa una gran satisfacción de parte de la gente con el tamaño-confort del hábitat. El diseño del hábitat en el centro de Montreal toma seriamente al aspecto del tamaño del espacio que se construye con objeto de evitar una caída (espacios no funcionales) en el confort, lo cual impactaría muy negativamente al mercado de la vivienda local ya sea en venta o en renta. 
Así el intercambio o "juego" (trade off) implícito entre las variables menor tamañoconfort y mayor accesibilidad al centro que la teoría geográfica económica proclama en sus modelos de ubicación (locational models) (Ross et ál, 2000 y Alonso, 1964), así como, la distinta composición de los hogares, ha sido eficientemente manejado en la ciudad de Montreal (notando que no siempre un menor o mayor tamaño de vivienda conduce a un menor o mayor confort, sino mas bien la relación que dicho tamaño menor o mayor mantenga con su número de usuarios, más crítico en familias más grandes).

Por su parte, la satisfacción con el mantenimiento de la vivienda en relación al confort es reportada por el público canadiense como muy significativa. El índice que se obtuvo de 0,85 en la tabla 3 y gráfico 1 , es representativo de esta situación.

En el mundo real, el esmero por el cuidado con el que la gente (en especial los propietarios) le dedica al factor mantenimiento de las propiedades se pone de manifiesto una vez más por razones no solo de tipo estético y personal, sino mas bien de tipo reglamentario. Los reglamentos que el gobierno impone son estrictos en la ciudad, en relación al estado de las viviendas, principalmente si estas se dedican al mercado de las rentas, el cual es muy importante en el centro.

En el presente análisis, la correlación de resultados ahora en términos de la importancia residencial es también muy representativa. En el perímetro II, rubro vivienda se hace evidente la importancia que los residentes le otorgan a la condición de las propiedades en función de su mantenimiento. Con un índice de 0,95 en la tabla 3 y gráfico 2, los habitantes del área explican en esa medida el estado actual de la vivienda local según el grado de importancia por su mantenimiento.

Desde el punto de vista de los reglamentos municipales vigentes que como anteriormente se aclaró son más estrictos, los habitantes de este perímetro se esfuerzan por conservar las propiedades funcionando lo más aceptable posible. Enfrentando condiciones climáticas a veces realmente difíciles (acumulación de cantidades significativas de nieve, hielo, etc., sobre las estructuras durante el largo invierno canadiense), la gente es forzada a poner mucha atención al asunto del mantenimiento.

La correlación importancia por tamañoimportancia por confort confirma por su parte lo trascendental que son esos dos conceptos de vivienda para los residentes en cuestión. A través del índice de 0,83 , la gente expresa en la tabla 3 y gráfico 2 su preocupación por estos, informando que un tamaño adecuado de vivienda que cumpla con las necesidades de espacio tanto individuales como colectivas de la familia, favorece al confort.

Adicionalmente, una relación equilibrada tamaño-confort incrementa la rentabilidad de la propiedad, lo que se traduce en una mayor estabilidad en el mercado local de vivienda, menores cambios de propietarios/inquilinos $y$ mayor seguridad en lo que respecta a ingresos municipales.

La importancia mantenimiento-confort (tabla 3, gráfico 2) es significativa en el centro de la ciudad. El índice de 0,92 indica una muy alta correlación entre estas dos variables. Las respuestas obtenidas durante la investigación de campo confirman en ese orden, la "cultura inmobiliaria" heredada e impulsada por el gobierno en relación a la atención por el hábitat $y$ medio ambiente de la ciudad. Esta situación es un hecho comprobable a través de la producción de una serie de ciudades bien organizadas en Canadá.

El mayor (y mejor) mantenimiento del contexto urbano primario se ha traducido para esta sociedad en un mayor confort, lo que ha beneficiado la re-densificación central que el gobierno se esfuerza en promover.

Continuando con los resultados de esta sección en el contexto urbano secundario, misma área geográfica, rubro de sitios de reunión, la población residente manifiesta la gran satisfacción que siente con los lugares socioculturales y con los lugares recreativos disponibles en dicha área (tabla 3, gráfico 1). El alto índice de correlación de 0,99 que sus respuestas produjeron hace evidente la adecuada dotación de este tipo de instalaciones en el perímetro II para actividades de esparcimiento popular. 
Cuando la gente tiene la posibilidad de enriquecer su calidad de vida con actividades de este tipo, aumenta la satisfacción residencial con el entorno. Una mayor satisfacción produce una mayor voluntad para continuar residiendo libremente en el perímetro que se menciona, incrementando con esto eventualmente el nivel de atracción del mismo para reforzar la optimización de los espacios centrales y últimamente, su sustentabilidad.

Finalmente, en el rubro servicios públicos, la correlación entre la importancia por centros de ayuda social con la importancia por hospitales, es otra vez alta (0,93 en la tabla 3 y gráfico 2). El índice obtenido es un reflejo directo de la alta consideración que los locales le otorgan a esas variables, lo que a su vez impacta tanto a la cantidad como a la calidad de las instalaciones disponibles en el área que ellos exigen.

En función de la respuesta que den las autoridades a esa exigencia, se encuentra la mayor/menor voluntad de permanecer libremente residiendo en el perímetro en cuestión o bien, de mudarse a otro lado, determinando esto la optimización o no del uso del espacio central que como se ha dicho, el gobierno busca fomentar en alguno de los programas de renovación urbana concebidos para la Ciudad de Montreal.

\section{GRÁFICO 2 \\ CORRELACIÓN RESIDENCIAL MONTREAL}

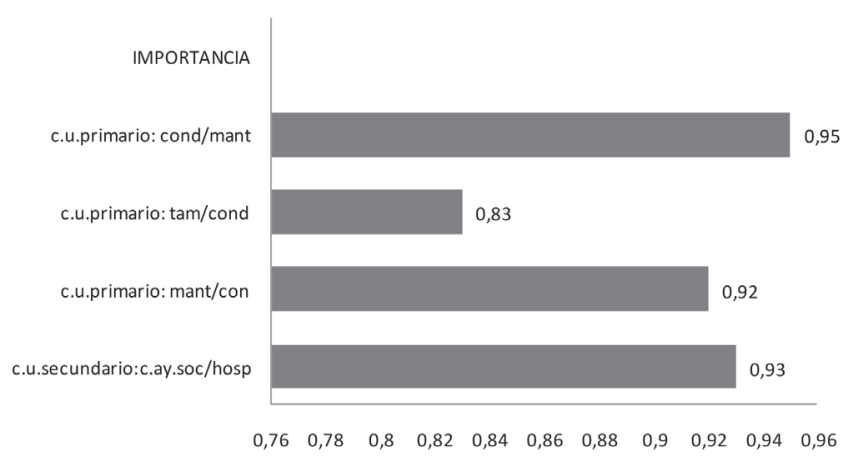

Fuente: Elaboración propia.

\section{CONCLUSIÓN}

Frente al problema del abandono central y la incontrolable expansión periférica, Montreal ha intentado llegar a un equilibrio de crecimiento más sustentable. Las acciones de re-densificación implementadas han logrado mejores resultados a pesar de la "complicidad capitalista" que en los centros históricos, arte y real estate realizan según la opinión de algunos autores neo-marxistas (Smith, 1996). Los centros necesitan re-densificarse, por lo cual, si es necesario, deberá usarse el capital para impulsar y mejorar los programas de renovación. En función de esos programas de renovación y consecuentes correlaciones presentadas, se afirma que la centralidad primaria de la ciudad de Montreal sustancialmente difiere en la forma de percibir el aspecto de la satisfacción residencial. En el caso del Municipio de Montreal, la aplicación de los distintos programas de renovación urbana ha producido índices de correlación más consistentes. Los canadienses que habitan el centro-perímetro II tradicionalmente han obtenido (a través de los programas descritos) en cantidad y calidad de vivienda y servicios, una 
más oportuna atención a sus necesidades de habitación entre otras, lo cual se puede aplicar a otros casos.

Ese panorama abre las puertas para que los organismos encargados continúen monitoreando el grado de eficiencia terminal (satisfacción residencial) de sus programas de renovación en turno para así garantizar en lo posible, su mayor adecuación a la realidad que intentan mejorar: la re-densificación del centro. Esto a su vez, redundará en desarrollos urbanos centro-periferia más equilibrados y compactos. La consistencia de índices que se obtuvo confirma esa adecuación. Su sociedad es culturalmente más meticulosa en muchos aspectos de la vida diaria como lo es la atención y cuidado por el medio ambiente, sea este el construido por el hombre (ciudades) o el natural. Canadá se ha estructurado a través de una serie de ciudades consideradas como planificadas y "limpias" (con menores problemas de contaminación ambiental que otros países) y realiza un esfuerzo constante por mejorar ese orden.

Como consecuencia de los objetivos de investigación planteados en este estudio, la formulación de las preguntas de investigación encuentra una respuesta positiva en las mejores correlaciones de Montreal que alientan una atracción urbana más significativa. Los resultados ofrecen una razonable esperanza de que los programas de renovación con la eficiencia actual, algún día contribuirán a lograr un crecimiento más equilibrado (compacto) centro-periferia que este trabajo promueve (re-densificación central), tan necesario para muchas ciudades de América. Esta esperanza se siente aún un poco lejana, dado lo difícil de recuperar las bases demográficas originales como la del centro de Montreal de 1971 y los importantes crecimientos periféricos que muchos casos todavía reportan.

Respecto a la validación de la hipótesis de investigación, esta queda confirmada parcialmente por el perímetro II del Municipio de Montreal, debido a que sus programas de renovación han resultado ser eficientes; no obstante, estos todavía podrían mejorar los índices mostrados de satisfacción residencial, los cuales motiven a las personas a continuar habitando voluntariamente el centro de la ciudad.

Es en función del cada vez mayor preocupante desequilibrio demográfico centroperiferia, que muchas de las grandes ciudades todavía experimentan, en donde el propósito de este estudio se ubica en términos de contribución al conocimiento: investigar las causas de dicho desequilibrio desde la perspectiva de la satisfacción residencial, en la cual poco se conoce amalgamando lo cualitativo con lo cuantitativo para así intentar representar la realidad que se vive. En ese sentido, se espera que dicha investigación permita así una mejor comprensión de los efectos negativos del proceso demográfico citado para asistir a las distintas agencias de planificación en el diseño de políticas urbanas más eficientes, las cuales dirijan más acertadamente las acciones resultantes en términos de planes y programas que prevean los efectos mencionados. Esto conducirá a un monitoreo más fácil de la eficiencia terminal de los programas de renovación involucrados, cuyo resultado se traducirá en una más apropiada administración del desarrollo urbano desde el punto de vista de su sustentabilidad.

\section{BIBLIOGRAFÍA}

\section{LIBROS}

Alonso, William. Location and land use: toward a general theory of land rent. EeuU: Harvard University press, 1964.

Babbie, Earl Robert y Halley, Frederick. Adventures in social research. Data analysis using sPSS. California, Eeuv: Pine Forge press, 1993.

Bourne, Larry; Hutton, Tom; Shearmur, Richard y Simmons, Jim. Canadian Urban Regions. Trajectories of Growth and Change. Ontario, Canada: Oxford University Press, 2011.

Germain, Annick y Rose, Damaris. Montreal: the quest for a metropolis. United Kingdom: John Wiley and Sons Limited, 2000.

Rose, Damaris. The uneasy cohabitation of gentrification and 'social mix': a case 
of residents of infill condominiums in Montreal. Quebec, Canadá: INRSUrbanisation, 2004.

Roseland, Marc. Le développement durable des centres urbains: guide à l'intention des pouvoirs locaux et municipaux. Ontario, Canadá: 1992.

Ross, Simon; Morgan, John y Heelas, Richard. Essential as geography. Nelson Thornes Ltd, 2000.

Roy, Jean Hugues y Weston, Brendan. Montreal. A citizen's guide to politics. Montreal, Quebec: Black Rose Books Limited, 1990.

Smith, Neil. The new urban frontier: gentrification and the revanchist city. London: Routledge, 1996.

\section{PUBLICACIONES PERIÓDICAS}

Affleck, Gavin. "Renewing the urban fabric: social housing in Montreal". The canadian architect 7 (49). Canadá. Canadian Architect, 2004: 17-18. En: <http://www. canadianarchitect.com/news/renewingthe-urban-fabric-social-housing-inmontreal/1000157100/> [consultado el 18 de agosto de 2013].

Bunting, Trudy; Filion, Pierre y Priston, Heath. "Changing patterns of residential centrality: population and household shift in large Canadian cmas, 1971-1996". Cahiers de Géographie du Québec 123(44). Canadá. Érudit, 2000: 341361. En: <http://www.erudit.org/revue/ cgq/2000/v44/n123/022925ar.pdf> [consultado el 14 de julio de 2013].

Castells, Manuel. "Le centre urbain. Projet de recherche sociologique". Cahiers internationaux de Sociologie 46. Francia. Presses Universitaires de France, 1969: 83-106. En: <http://www. jstor.org/stable/40689481> [consultado el 10 de octubre de 2013].

Chantal, Suzanne y Charbonneau, Stéphane. "Le rôle de l'évaluation foncière au service d'une approche territoriale ciblée: le cas de Montréal". Revue Urbanité. Canadá. Canadian Institute of Planners, 2004:1-2. En: <http://ville.montreal. qc.ca/pls/portal/docs/page/habiter_v2_fr/ media/documents/urbanite_final.pdf> [consultado el 30 de agosto de 2013].

Dansereau, Francine; Charbonneau, Stéphane; Morin, Richard; Revillard, Anne; Rose, Damaris y Seguin, Anne-Marie. "La mixité social en habitation". Recherche pour la Direction de l'Habitation de la Ville de Montréal. 2002:151. En: <http:// www.clic-bc.ca/Documents/E-CtreDoc/ Cadre-de-vie/Logement/ca\% mixite $\% 20$ sociale $\% 20$ en $\% 20$ habitation.pdf $>$ [consultado el 3 de enero de 2014].

Sénecal, Gilles. "Champs urbains et développement durable: les approches Canadiennes de la ville écologique". Natures, Sciences, Sociétés 1(4). Francia. Asociación Naturalezas Ciencias Sociedad-Diálogo, 1996: 61-74.

Tello, Carlos Alberto. "Regeneración y calidad de vida urbana en el centro antiguo de las Ciudades de Montreal y México: una comparación”. Economía, Sociedad y Territorio 14 (13). Toluca, México. El Colegio Mexiquense, 2013: 123.

Wilson Vanvoorhis, Carmen y Morgan, Betsy. "Understanding power and rules of thumb for determining simple sizes". Tutoring in Quantitative Methods for Psychology 2(3). eeuu. University of Wisconsin-la Crosse, 2007.

Wright, Tommy. "Exact Confidence Bounds When Sampling from Small Finite Universes". Lecture Notes in Statistics 66. Berlin, Germany. Springer-Verlag, 1991.

\section{TEXTOS ELECTRÓNICOS}

Canada Mortgage and Housing Corporation (смнс). Portrait de l'activité de la construction dans la Région Métropolitaine de Montréal. 2004. En: $<w w w . c m h c-s c h l . g c . c a>$ [consultado el 15 de septiembre de 2013].

Chantal, Suzanne. "Investir à Montréal". Service de la mise en valeur $d u$ territoire et du patrimoine, direction de l'habitation de la Ville de Montréal. 2004: 7. En: <http://ville.montreal.qc.ca/pls/ 
portal/docs/page/habiter_v2_fr/media/ documents/suz_cha.pdf> [consultado el 20 de agosto de 2013].

Demographia. World Urban Areas and Population Projections. Fifth annual edition, abril 2009. En: <www. demographia.com/db-worldua.pdf $>$ [consultado el 2 de julio de 2013].

Dansereau, Francine; Charbonneau, Stéphane; Morin, Richard; Revillard, Anne; Rose, Damaris y Séguin, Anne-Marie. La mixité social en habitation. 2001. En: < http://www.vrm.ca/documents/ dansereau\&al2002-03.pdf > [consultado el 3 de enero de 2014].

Société d'habitation du Québec. Programmes et services Accès Logis Québec. 2005. En: <www.habitation.gouv.qc.ca/ programmes/accèslogis $>$ [consultado el 1 de septiembre de 2013].

Solomon, Lawrence. How to save the farm: more immiggration, less suburbanization. Toronto, Canada: The Urban Renaissance Institute, 2000. En: <http://www.urbanrenaissance. probeinternational.org/2000/02/08/ how-save-farm-more-immigration-lesssuburbanization/> [consultado el 4 de julio de 2013]

Statistics Canada. Census of Canada Database. 1971. Ministry of Industry, Science and Technology. En: <http://www.statcan. $\mathrm{ca} />$ [consultado el 4 de julio de 2013].

Statistics Canada. Census of Canada Database. 1981. Ministry of Industry, Science and Technology. En: <http://www.statcan. $\mathrm{ca} />$ [consultado el 6 de julio de 2013].

Statistics Canada. Census of Canada Database. 1986. Ministry of Industry, Science and Technology. En: <http://www.statcan. $\mathrm{ca} />$ [consultado el 16 de septiembre de 2013].

Statistics Canada. Census of Canada Database. 1991. Ministry of Industry, Science and Technology. En: <http://www.statcan. $\mathrm{ca} />$ [consultado el 8 de julio de 2013].

Statistics Canada. Census of Canada Database. 2001. Ministry of Industry, Science and Technology. En: <http://www.statcan. $\mathrm{ca} />$ [consultado el 14 de julio de 2013].

Statistics Canada. Census of Canada Database. 2006. Ministry of Industry, Science and Technology. En: <http://www.statcan. gc.ca/> [consultado el 20 de septiembre de 2013].

Organización de las Naciones Unidas. World Population Prospects. Department of Economic and Social Affairs, Population Division. EeuU. 2003. En: <www. unpopulation.org $>$ [consultado el 6 de julio de 2013].

Ville de Montréal. Programmes en vigueur pour la création de logements sociaux et communautaires à Montréal, Année 2004. Service de la Mise en Valeur du Territoire et du Patrimoine. Direction de la Planification Stratégique. Division de la Planification de l'Habitation. 2005. En: <http://arrondissement.com/toutlist-document/s40-logement-social> [consultado el 18 de agosto de 2013].

Ville de Montréal. Habiter Montréal. Direction de l'Habitation de la Ville de Montréal, Service de la Mise en Valeur du Territoire et du Patrimoine. Canadá. 2002. En: $<$ http://ville.montreal.qc.ca> [consultado el 15 de octubre de 2013].

TESIS

Tello, Carlos Alberto. "Revitalización urbana y calidad de vida en el sector central de las Ciudades de Montreal y México". [Tesis de Doctorado en Geografía Urbana]. Universidad Nacional Autónoma de México (unam), 2009.

OTROS

Tello, Carlos Alberto. Montreal. "Residential rehabilitation in downtown, 19611996". [Research report]. Department of Geography, School of Graduate Studies and Research, University of Ottawa, 1997: 77.

Tello, Carlos Alberto. "Extrapolaciones de Población para el Sector Central de las Ciudades de Montreal y México". [Reporte de investigación]. Universidad 
Nacional Autónoma de México (unam), Facultad de Filosofía, Departamento de Geografía. 2008.

United Nations. Habitat II. Canadian National Report. United Nations Conference on Human Settlements. Istanbul, Turkey, 1996.

United Nations. World Population Prospects.

New York, Estados Unidos: United
Nations-Department of Economic and Social Affairs, Population Division, 2003. En: <http://www.unpopulation.org> [consultado el 6 de julio de 2013].

Fecha de ingreso: 12/02/2014 Fecha de aprobación: 01/07/2014 
